\title{
Municipal Engineer: Referees 2019
}

The following is a list of referees who have reviewed papers for Municipal Engineer between 1 December 2018 and 30 November 2019. The Institution of Civil Engineers is very grateful for their assistance.

We are continually looking for suitable reviewers for papers submitted to Municipal Engineer. Papers published in the Proceedings of the ICE must be submitted to at least two independent referees to judge accuracy, style, impact, importance and interest.

If you are interested in reviewing articles on any topic related to municipal engineer, please submit your name, qualifications or CV,

Jitendra Agarwal

Kayvan Aghabayk

Gülçağ ALBAYRAK

Arminda Almeida

Robin Andrew

Ali Ataie

Sana Balouch

Jonathan Bateman

Simon Bowers

Amit Chanan

Xi Chen

Ricardo Nuno Francisco do Carmo

A. A. Elziny

Marcus Enoch
Cidália Fonte

Jonathan Foster-Clark

Koorosh Gharehbaghi

Parviz Ghoddousi

Anne Gibbs

Peter Hallsworth

Richard Hanley

gareth hearn

E. Hellawell

Safak Hengirmen Tercan

Solmaz Hosseinioon

Neil Humphrey

R. Huxford

lan Jenkinson and areas of expertise. We are in need of individuals who will agree to review papers in a timely fashion (within 3 to 4 weeks of receipt) and provide confidential feedback to the Editorial Advisory Panel concerning the quality of the paper and any suggested revisions that would be appropriate.

If you are such a person, please contact Rebecca Rivers (tel.: +44 (0)207 665 2241; e-mail: rebecca.rivers@icepublishing.com) for more information on the referee process.

Sivakumar Kandasam

Murat Karacasu

Leena Korkiala-Tanttu

Seungjae Lee

Richard Little

José Paulo Pereira Gouveia Lopes

de Almeida

Rui Marques

Dinesh Mehta

Odair Moraes

Graham Newson

Andrew Otto

Zahra Pezeshki

Enrico Quagliarini
M. Rosário Ramos

Murray Reid

Anabela Ribeiro

Mohamed Salheen

Pedro Simao

Nuno Sousa

Joaquim Sousa

Emily Tan

John Thomson

Tom van Vuren

Pete Walker

Ben Waterson

Yong Yuan 